\title{
FILMING UNDERWATER IN 3D RESPECTING STEREOGRAPHIC RULES
}

\author{
R. Rinaldi ${ }^{a}, H$. Hordosch $^{b}$ \\ ${ }^{a}$ Underwater Photography, Rome, Italy - rinarobe@gmail.com \\ ${ }^{\mathrm{b}}$ Seacam - Cinema of Dreams, Voitsberg, Austria - office@ seacam.com
}

\section{Commission V}

KEY WORDS: Underwater, Photography, Stereoscopic 3D

\begin{abstract}
:
After an experimental phase of many years, 3D filming is now effective and successful. Improvements are still possible, but the film industry achieved memorable success on 3D movie's box offices due to the overall quality of its products. Special environments such as space ("Gravity") and the underwater realm look perfect to be reproduced in 3D. "Filming in space" was possible in "Gravity" using special effects and computer graphic. The underwater realm is still difficult to be handled. Underwater filming in 3D was not that easy and effective as filming in 2D, since not long ago. After almost 3 years of research, a French, Austrian and Italian team realized a perfect tool to film underwater, in 3D, without any constrains. This allows filmmakers to bring the audience deep inside an environment where they most probably will never have the chance to be.
\end{abstract}

\section{INTRODUCTION}

Today, this tool has been used for many, many dives in different locations around the world, but also in Mediterranean. It is filming in 3D in Mediterranean that brought a very big and surprising success. In the past, in fact, many times the audience was giving a big preference to documentaries showing the beautiful and colourful "tropical waters", in comparisons with the Med sea. Showing images of our sea in 3D totally changed the attitude and always is source of successful screenings. It is for this reason that more and more Marine parks, museums, schools are demanding 3D movies to be showed, declaring that they represent a true attraction. We are now sure that the $3 \mathrm{D}$ technique will be a unique tool to attract people to the knowledge and the conservation of the sea. And that it will be a great tool for education.

This is why we care to present some short movies about our seas. In $3 \mathrm{D}$, of course!

\section{DEFINITION}

To produce an image that gives the 3D effect, we need to use two cameras. This is a well known fact. Every camera represents one eye. In the human view, every eye sees an images just a little bit different from the one that the other eye sees. Part of the images coming from the two eyes is the same. Of course, the right eye will see more on the right side, and the same will happen to the left eye. 3D photography aims to reproduce this situation. This is true from a theorical and geometrical point of view. The concept is very easy: we need to shoot the same scene with two cameras. Of course, these cameras must be equipped with exactly the same lenses, and the settings must be exactly the same. The two cameras must be - like in the human view set one near by the other and they must reproduce two images covering a common part of the scene. Than, to be able to enjoy the tri dimensional view, we have to operate in the way that the right eye will see only the image shot by the right cam and the left eye must see only the image shot from the left eye.

Clear and easy the concept, difficult and complicate to make it real. Several questions are immediately coming when shooting: "we must produce two different images" ... what does mean "different"? Cameras have to be separated. Good! But how much? To try to solve these problems, we come back to observe the human view. Immediately, we'll notice that the eyes have a fix distance between the two of them and that this distance is between 5 and 10 centimetres.

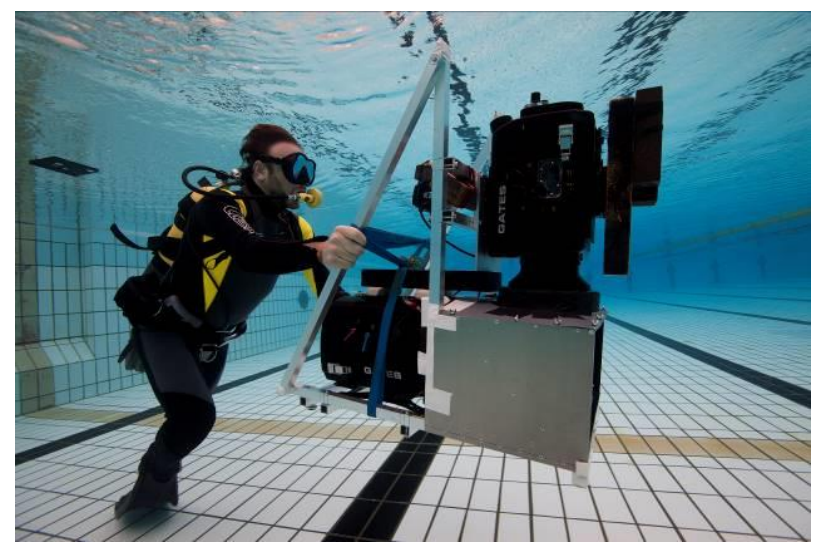

Figure 1. The first prototype: we were looking for using a wide angle on a beam splitter system. The idea was to use two housings equipped with dome and wide angle lens on a wet mirror box. The system was perfectly working in crystal clear waters. As soon as a particle was setting on the mirror, it was appearing only on one of the eyes. And this was destroying the possibility to use the sequence

We would like for this reason to say that we'll separate the two lenses of this distance and everything will work. This looks to be a very logical way to proceed, but will lead us to a complete wrong direction. To make this concept clear, I would like to come out from science, geometry and mathematics, and try to explain the situation using simple considerations. Simple, but representing the key of the problem. It is true that we can see in 3D. It is true that the eyes cannot change the distance in between themselves. But it is also true, that when we "watch" we concentrate on a subject. If we are sitting somewhere and we have a landscape in front of us, we will enjoy that view. Grass, trees, birds flying, flowers, clouds in the sky. We are enjoying the general view and every objet has his volume. Than, we collect a flower and we bring it very close to our eyes and watch it. We see it in 3D. Let's try to do this with a camera, now. We set two cameras side by side and shoot the land scape. Good, we 
probably will be able to get a 3D image. Than, we take a flower and we put it very close to the cameras. We'll immediately discover that one lens frames it, but the flower does not appear at all in the other camera. In the same time, the back ground, remains the same as it was before. This example to make clear that our brain is able to focus and concentrate on a subject and work on it to give it the right volume. Than, when the subject changes, the brain makes a new adjustment. Basically, when the brain is concentrate on one subject, it makes the right adjustments to see it, and all the rest will not be considerate. This is something that is simply impossible when filming or photographing. The stereographer must produce an image where all the subjects are right from a stereographical point of view. Because some of the audience is maybe interested to the flower in foreground, others may be interested to the background or the general view. So, the brain will not need to adjust: everything must be correct from a stereographical point of view. Without going deep in the matter, we already have understood that somehow the right inter axe is function of the distance between the cameras and the subject: the example of the flower very close tells us that if a subject is so near to the lenses, they must be almost overlapped to see it; framing mountains very far, we'll notice that if the cameras are some centimetres apart, the images are almost identical. This means no stereo effect or - at least - a very weak one. The stereographer, will be for this reason obliged to change the inter axe and the convergence at every take. The stereographer will increase or decrease the inter-axe value, depending from the distance between lens and subject. He will also be able to play with the convergence between the two cameras. Doing that, there will be for sure a point in the space where happens that the items framed from the two cameras are coincident. The two images are not separated at all. The objects which are in this situation, will be seen as they are exactly on the same level of the screen. Everything is closer to the cameras, will give the impression to be out of the screen - in the room - all is behind, will look as it is somewhere behind. It is from this point that another series of difficulties are coming out: we should think about the item closest to the camera and to the one which is the last in the far. The stereographer will need to calculate the maximum value of separation between the two images of the nearest object ( negative parallax ) and the same with the last in the far ( positive parallax ). The sum of the two values is what we call "depth budget". This is the value that the stereographer must keep in count when he built his dimension to the image. The depth-budget does not exceed a limited value, other wise, the image will be annoying for the audience, as the brain will not be able to make a natural synthesis between the two images. Much more should be said about this matter, but basically, this is the concept around we can work.

During last years, the willing of producing a "too strong" stereography, or the willing to use as an effect a too strong "negative parallax", or a series of mistakes in the geometry of the two images, produced headache, sour eyes or other discomfort in the audience that was the main reason of the lost of passion for the 3D. Producing a 3D film, means to be rigorous and to apply geometrical and mathematical rules, beside the artistic aspect. Therefore when first we have approached the idea of producing a 3D system for underwater use, we put this aspect over all the others: our goal was to produce underwater a perfect stereography, or not to produce $3 \mathrm{D}$ movies at all. No compromises on that.

\section{UNDERWATER FILMING}

Before facing that challenge, we made some general considerations about underwater filming. The first one is related to the water it self: everyone knows that water is more dense than air and that causes a lot of troubles in the vision. No one will ever think to watch the underwater world without wearing a mask. When we'll wear a mask, all objects will look closer and bigger than they are. This is due to the refraction that reduces our angle of view. It is like if we go in the water with a wide angle, and we see trough a tele-lens. This is not the only effect: due to its density, the water is less transparent than the air. I use to say that a foggy day with $50 \mathrm{mt}$ of visibility on land is a night mare for everyone, while a day with 50 metres of visibility underwater is a dream that an underwater photographer experience a few times in his life.

Combining the two effects together, all problems for an underwater photographer are coming out: the water is "turbid", so we need to be close to the subject to get sharp images. There fore we need a wide angle to be able to frame a significant portion of the underwater world. But the transmission between air and water, causes a significant loss on the angle of vision of our lens. To solve this problem, we can use a dome port in front of the lens. It is a spherical lens that does not bent the rays and that gives back to the lens its original characteristics.

\section{SETUP}

With this elements, let's come back to our 3D system and let's set some basic considerations:

- We must be as close as possible to the subject

- Therefore, our inter-axe must be really small

- A side by side system will be impossible to be used

- We must built a "beam splitter" system: the two cameras are perpendicular between them selves and a $45^{\circ}$ mirror is between them

- This will oblige us to have a very big housing to contain all that (Figure 2)

- To produce a good stereography, we must keep in count the depth budget. This means that we need a 3D assist monitor that allow us to overlap the images and to calculate the amount of stereography we have

- This monitor has to be housed, as well

- We must use wide angle lenses to be close enough to the subjects

- Our wide angle behind a flat port will lose its angle

- The basic consideration to be done to make a dome port work, is to set the centre of the sphere in the same position of the centre of the lens. This is impossible with two lenses and one sphere....

- This is the main point and it is on this point that our major efforts must be directed.

Reading our target list, at that time it was easy to notice that some of the goals have already been hit. Most of the major productions have built housings with beam splitter system. In the same way, very few people thought not to use a 3D assist monitor (Figure 3) for a stereographic movie. The main points that have been not solved, yet, were the size of the system and the port in front of the lenses. 


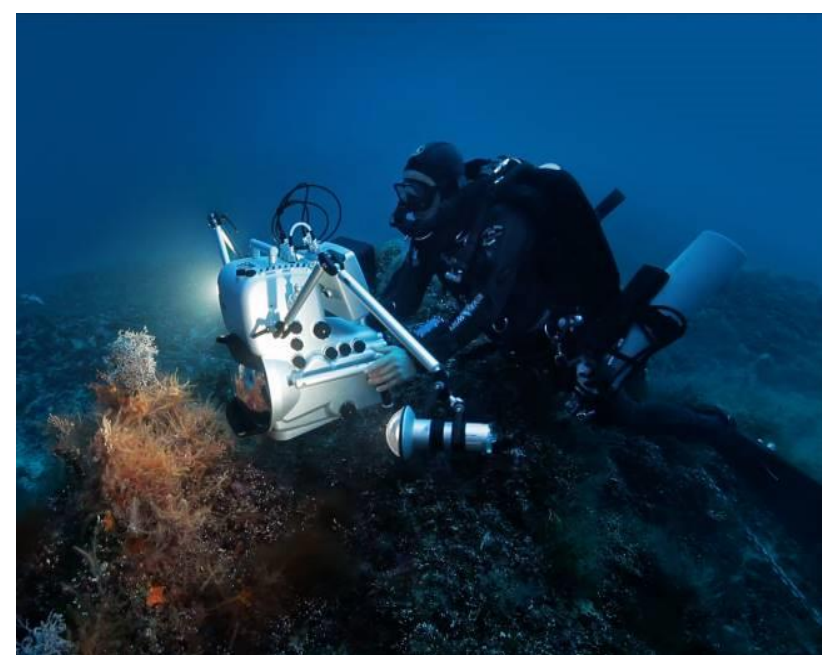

Figure 2. The housing used in Mediterranean: as it is possible to see and notice, the size is very small and the operator can work in the same time as cameraman and stereographer

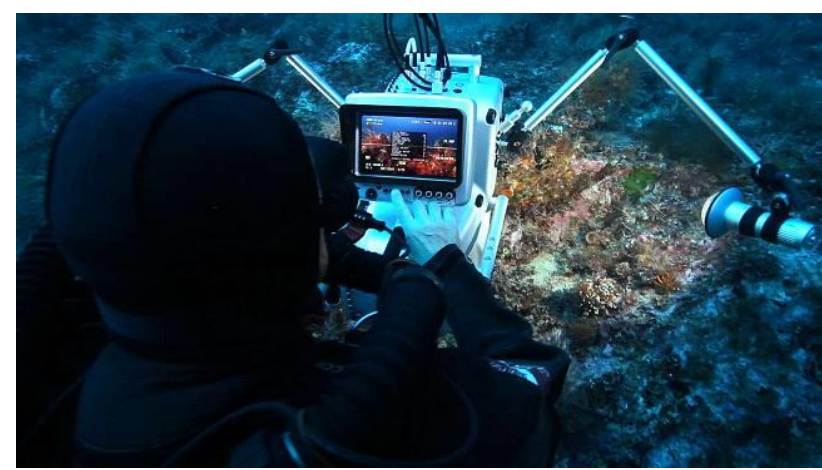

Figure 3. First tests at sea. At over 60 meters deep in Mediterranean, the operator is working on the 3D assist monitor: this is fundamental to achieve a good stereography. This monitor allows us to calculate the effect on a digital base while we are adjusting the inter-axe in real time

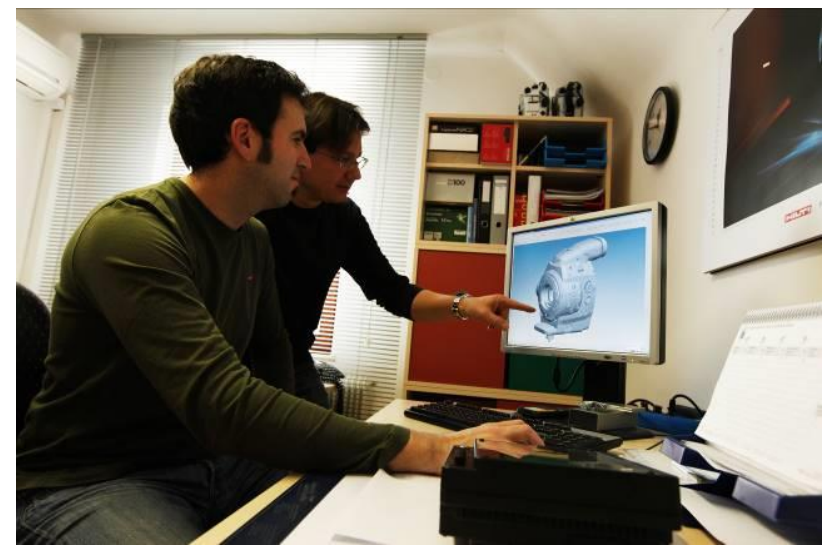

Figure 4. Harald Hordosh and his engineer Gunter Rupreckter started their project with a 3D scan of the cameras to be housed.

In this way all space was rationalized and the final size was amazing small

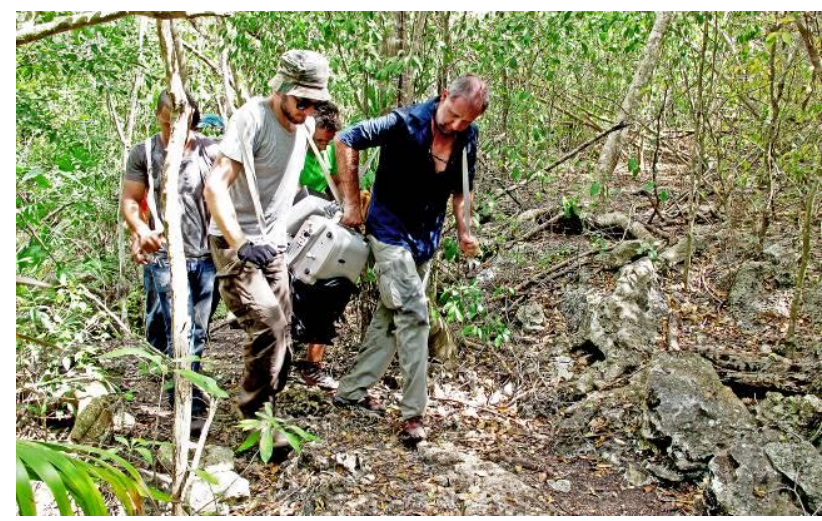

Figure 5. Big but no so much: a bit difficult but still possible to go to film a cenote in the meddle of Cuban forest, very far from the road.

Working on the size was easy: SEACAM is a factory specialized in underwater housings. It was easy for them to produce a project of an underwater stereographic system having a small size (Figure 4/5).

The key point was to think in a different way: everyone before was trying to bring underwater something that was built to work on land. Most of them get a complete stereographic rig and built an housing around. All that was not necessary. Underwater the visibility is less than on air, we have said that. Therefore, we were sure that a big inter axe was not needed. This allowed us to built a small mirror box and a smaller rig. We did not put a rig inside and housing, but SEACAM engineers used the housing as structure for the stereo rig. The final result was an housing not bigger than an old housing for a digital Betacam.

The real challenge was when we faced the issue of the optical problems. We mentioned already the effects of refraction when a lens is behind a flat glass. We said that we were going to lose angle of view and that our subjects were going to appear bigger and closer. We did not mention that the flat port affects the quality of the lenses. Both in chromatic and optical characteristics. The most evident issue is the lost of sharpness in the corner of the image. This is more true and evident with wider lenses. It is exactly what we do not want to experience when working underwater, in an environment where the wide angle is a must. Together with SEACAM engineers, we struggled for almost one year to find a solution.

Using my knowledge of over 30 years of photographing underwater, I was able to figure out a model that could work, thanks to some simple but clever ideas. I have spoken wit $\mathrm{Mr}$ Immanuel Hordosh about my concept, and he was able to calculate it and to put it on paper, drawing an optical scheme of the system made from a very large dome port, the mirror box and two lenses going from $14 \mathrm{~mm}$ to the zoom $16-35$, to the $50 \mathrm{~mm}$ macro lens. On the paper our theory was working. At that point, nothing else for us than accept or refuse to take the challenge of investing time and money to built our prototype. We did and started the construction. Always fearing to be on the wrong way. When finally ready, we tested the system inside the pool, and the results were stunning. Also using very open $f$ stops, the images did not have aberrations of any kind and the angle of the lens was maintained (Figure 6). 


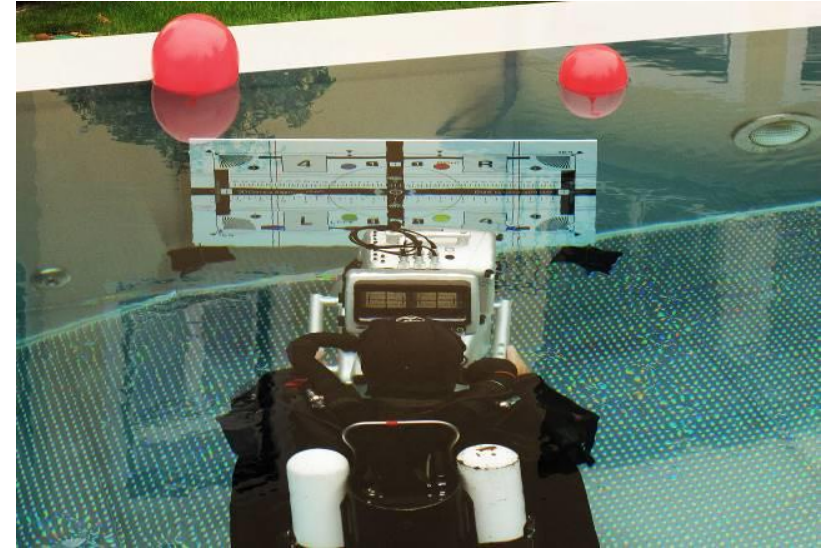

Figure 6 . The first tests we made in the pool were very accurate and severe: here we are filming a chart using $\mathrm{f} 2.8$ in order to be sure about the dome port quality

\section{CONCLUSION}

We created the first and unique underwater 3D system equipped with wide angle lens and a dome port to correct the refraction (Figure 7).

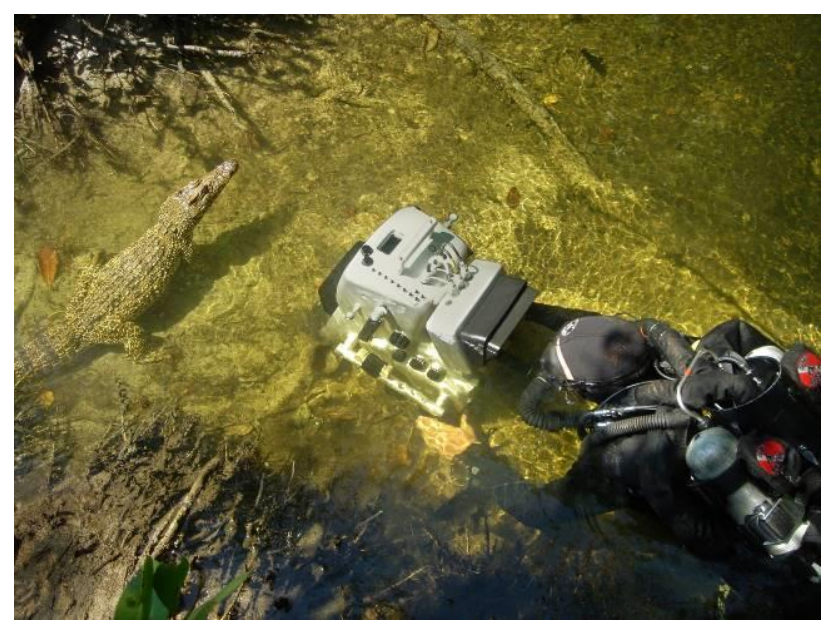

Figure 7. The system we produced is versatile and handily. It was possible to reach very remote environments and freely approach wild animals. As we can see in this image, also working in muddy waters (such as mangrove forest) was possible thanks to the dome port and the wide angle.

After that, I have used the system many times and for many movies. At least 4 documentaries of 52 minutes each for Discovery Channel have been produced, as well as a cinema movie produced by Paramount and Orange FR. Several short documentaries for big and medium screen have been realized for museums and marine parks.

Today our system is well known and appreciate in the film industry all over the world.

\section{REFERENCES}

Block, B., Mcnally, P., 2013. 3D Storytelling: How Stereoscopic 3D Works and How to Use It, Taylor \& Francis.
Czinkota, M., Ronkainen, I., 2012. International Marketing, Cengage Learning.

Fossati, G., 2009. From Grain to Pixel: The Archival Life of Film in Transition, Amsterdam University Press.

Haye, R. M., 1998. 3-D Movies: A History and Filmography of Stereoscopic Cinema, McFarland.

Mendiburu, B., 2011. 3D TV and 3D Cinema: Tools and Processes for Creative Stereoscopy, Taylor \& Francis.

Mendiburu, B., 2009. 3D Movie Making: Stereoscopic Digital Cinema from Script to Screen, Taylor \& Francis.

Pennington, A., Giardina, C., 2013. Exploring 3D: The New Grammar of Stereoscopic Filmmaking, CRC Press.

Peres, M. R., 2007. The Focal Encyclopedia of Photography: Digital Imaging, Theory and Applications, History, and Science, Taylor \& Francis.

Purse, L., 2013. Digital Imaging in Popular Cinema, Oxford University Press.

Saxby, G., 2013. The Science of Imaging, Second Edition, CRC Press.

Zone, R., 2012. 3-D Revolution: The History of Modern Stereoscopic Cinema, University Press of Kentucky. 